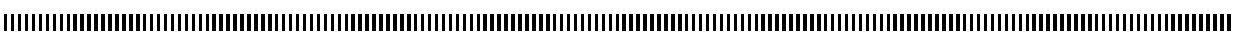
|

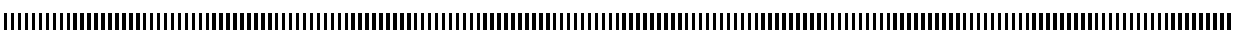
| | | |

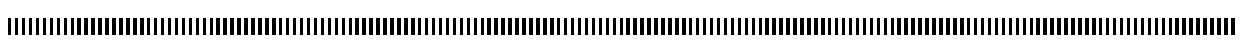

Rubrique

\title{
Inverse heat source problem for a coupled hyperbolic-parabolic system
}

\author{
Bellassoued Mourad and Riahi Bochra
}

LASMIN, University of Tunis El Manar, National engineering school of Tunis, ENIT, Tunisia, mourad.bellassoued@fsb.rnu.tn

Department of Mathematics, University of Carthage, Bizerte, Tunisia

riahi.bochra@gmail.com

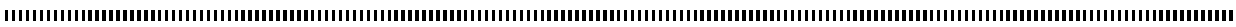

RÉSUMÉ. Dans ce papier, on a prouvé une estimation de stabilité de type Höldérienne pour un problème inverse de détermination du terme source de l'équation de la chaleur à l'aide d'une inégalité de Carleman pour un système d'équations hyperbolique-parabolique couplé.

ABSTRACT. In this paper we consider a coupled system of mixed hyperbolic-parabolic type which describes the Biot consolidation model in poro-elasticity. Using a local Carleman estimate for a coupled hyperbolic-parabolic system, we prove the uniqueness and a Hölder stability in determining the heat source by a single measurement of solution over $\omega \times(0, T)$, where $T>0$ is a sufficiently large time and a suitable subbdomain $\omega \subset \Omega$ such that $\partial \omega \supset \partial \Omega$.

MOTS-CLÉS : Problème inverse, estimation de Carleman, système couplet

KEYWORDS : Inverse problem, Carleman estimate, coupled system

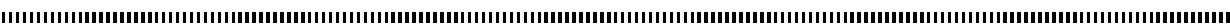




\section{Introduction}

Let $\Omega \subset \mathbb{R}^{3}$ an open and bounded domain with $\mathcal{C}^{\infty}$ boundary $\Gamma=\partial \Omega$, and let $t$ and $x=\left(x_{1}, x_{2}, x_{3}\right) \in \Omega$ denote the time variable and the spatial variable respectively. Given $T>0$, we consider a coupled hyperbolic-parabolic system

$$
\begin{cases}\mathbf{u}_{t t}-\Delta_{\mu, \lambda} \mathbf{u}-\nabla\left(\lambda^{*}(x) \operatorname{div} \mathbf{u}_{t}\right)+\varrho_{1}(x) \nabla \theta=0 & \text { in } Q \equiv \Omega \times(0, T) \\ \theta_{t}-\Delta \theta+\varrho_{2}(x) \operatorname{div} \mathbf{u}_{t}=g & \text { in } Q \\ \mathbf{u}(x, t)=0, \quad \theta(x, t)=0 & \text { on } \Sigma \equiv \Gamma \times(0, T)\end{cases}
$$

where the ${ }_{t}$ stands for the time derivative, $\nabla=\left(\partial_{1}, \partial_{2}, \partial_{3}\right)$, and $\Delta_{\mu, \lambda}$ is the elliptic second order linear differential operator given by

$$
\begin{aligned}
\Delta_{\mu, \lambda} \mathbf{v}(x) & \equiv \mu \Delta \mathbf{v}(x)+(\mu+\lambda)(\nabla(\operatorname{div} \mathbf{v}(x))) \\
& +\operatorname{div} \mathbf{v}(x) \nabla \lambda(x)+\left(\nabla \mathbf{v}+(\nabla \mathbf{v})^{T}\right) \nabla \mu(x), \quad x \in \Omega
\end{aligned}
$$

for $\mathbf{v}=\left(v_{1}, v_{2}, v_{3}\right)^{T}$, where.$^{T}$ denotes the transpose of matrices. Throughout this paper, $\mathbf{u}=\left(u_{1}, u_{2}, u_{3}\right)^{T}$ denotes the displacement at the location $x$ and the time $t$, and $\theta \equiv$ $\theta(x, t)$, the temperature, is a scalar function, $g \in H^{1}\left(0, T ; L^{2}(\Omega)\right)$ is a heat source. We will assume that the Lamé parameters $\mu, \lambda \in \mathcal{C}^{2}(\bar{\Omega})$, satisfy

$$
\mu(x) \geq \mu_{0}>0, \quad \lambda(x)+2 \mu(x)>0, \quad \forall x \in \bar{\Omega} .
$$

and $\lambda^{*} \in C^{2}(\bar{\Omega})$ is the consolidation coefficient which satisfy :

$$
\lambda^{*}(x) \geq k_{0}>0, \quad x \in \bar{\Omega} .
$$

Furthermore, the coupling coefficients $\varrho_{1}, \varrho_{2}$ satisfies :

$$
\left(\varrho_{1}, \varrho_{2}\right) \in\left(\mathcal{C}^{2}(\bar{\Omega})\right)^{2} ; \quad \varrho_{1}(x)>\varrho_{0}>0 \text { in for all } x \in \bar{\Omega} .
$$

We assume that the heat source is given by

$$
g(x, t)=q(x) k(x, t)
$$

where $k \in W^{2, \infty}(Q)$ and $q \in H^{2}(\Omega)$.

We can prove (e.g., $[1,5])$ that the system (1) possesses a unique solution

$$
(\mathbf{u}, \theta) \equiv\left(\mathbf{u}_{\left(\lambda^{*}, \varrho_{1}, \varrho_{2}\right)}, \theta_{\left(\lambda^{*}, \varrho_{1}, \varrho_{2}\right)}\right),
$$

Let $\omega \subset \Omega$ be a given arbitrarily subdomain such that $\partial \omega \supset \Gamma$, i.e. $\omega=\Omega \cap V$ where $V$ is a neighbourhood of $\Gamma$ on $\mathbb{R}^{3}$ and let $k$ and $t_{0} \in(0, T)$ be appropriately given.

Inverse Problem : Determine $q(x), x \in \Omega$, by measurements

$$
\left.\mathbf{u}\right|_{\omega \times(0, T)}, \quad \mathbf{u}\left(x, t_{0}\right), \quad \text { and } \theta\left(x, t_{0}\right) \quad x \in \Omega .
$$

The main subject of this paper is the inverse problem of determining of $g$, in the Biot consolidation model in poro-elasticity, uniquely from observed data of displacement vector $\mathbf{u}$ on a suitable subdomain $\omega \subset \Omega$ and the observation data of $\mathbf{u}$ and $\theta$ at given a 
suitable time $t_{0}$. Such kinds of observation data are similar to those considered in (e.g. [2], [3], [4]).

The key ingredient in our argument is an $L^{2}$-weighted inequality of Carleman type for coupled mixed hyperbolic-parabolic system. We prove a Hölder stability estimate in our inverse problem. We note that the uniqueness in the inverse problem follows directly from the Hölder stability.

\subsection{Statement of main result}

Let $t_{0} \in(0, T)$ and $x_{0} \in \mathbb{R}^{3} \backslash \bar{\Omega}$ such that

$$
\frac{1}{\sqrt{r_{0}}} \max _{x \in \bar{\Omega}}\left|x-x_{0}\right|<\min \left\{t_{0}, T-t_{0}\right\},
$$

where $r_{0} \in\left(0, \mu_{0}\right)$.

We denote $(\mathbf{u}, \theta)$ the solution of (1) corresponding to $\left(\varrho_{1}, \varrho, \lambda^{*}\right)$.

Theorem 1.1 Let $x_{0} \in \mathbb{R}^{3} \backslash \bar{\Omega}$ and $t_{0} \in(0, T)$ satisfies (4).

Let $k \in W^{2, \infty}(Q)$ such that $\|k\|_{W^{2, \infty}} \leq M$ and

$$
k\left(x, t_{0}\right) \neq 0, \quad x \in \bar{\Omega} .
$$

We assume that the solution $(\mathbf{u}, \theta)$ satisfies the a priori boundedeness :

$$
\|\mathbf{u}\|_{H^{2}\left(0, T ; H^{2}(\Omega)\right)}+\|\theta\|_{H^{1}\left(0, T ; L^{2}(\Omega)\right)} \leq M_{0},
$$

for some given positive constant $M_{0}$.

Then there exist constants $C>0$ and $\delta \in(0,1)$ such that the following stability estimate hold

$\|q(x)\|_{L^{2}(\Omega)} \leq C\left(\left\|\mathbf{u}_{t}\right\|_{H^{4}(\omega \times(0, T))}+\left\|\theta\left(., t_{0}\right)\right\|_{H^{2}(\Omega)}+\left\|\mathbf{u}_{t}\left(., t_{0}\right)\right\|_{H^{3}(\Omega)}+\left\|\mathbf{u}_{t t}\left(., t_{0}\right)\right\|_{H^{1}(\Omega)}\right)^{\delta}$.

By Theorem 1.1, we can readily derive the uniqueness in the inverse problem

Corollary 1.2 Under the same assumptions as in Theorem 1.1, we have the uniqueness. Let $(\mathbf{u}, \theta)$ satisfy the Biot system (1) such that

$$
\mathbf{u}(x, t)=0, \quad(x, t) \in \omega \times(0, T)
$$

and

$$
\mathbf{u}\left(x, t_{0}\right)=0, \quad \theta\left(x, t_{0}\right)=0, \quad x \in \Omega .
$$

Then

$$
q(x)=0 \text { for all } x \in \Omega .
$$

The remainder of the paper is organized as follows. In section 2, we give a Carleman estimate for a coupled hyperbolic-parabolic system. In section 3, we prove Theorem 1.1. 


\section{Carleman estimate}

Here we present a Carleman estimate, which was proved in [3]. For formulating our Carleman estimate, we introduce some notations. Let $\psi: \Omega \times \mathbb{R} \longrightarrow \mathbb{R}$ by setting

$$
\psi(x, t)=\vartheta(x)-\beta\left(\left(t-t_{0}\right)^{2}-M\right)=\left|x-x_{0}\right|^{2}-\beta\left(\left(t-t_{0}\right)^{2}-M\right), \quad x \in \bar{\Omega},
$$

for $M>0$ large, where $x_{0} \in \mathbb{R}^{3} \backslash \bar{\Omega}$ and $t_{0} \in(0, T)$ such that

$$
\min \left\{t_{0}^{2},\left(T-t_{0}\right)^{2}\right\}>r_{0}^{-1}\left(\max _{x \in \bar{\Omega}} \vartheta(x)\right) .
$$

We fix $\delta>0$ and $\beta>0$ satisfying

$$
\beta \min \left\{t_{0}^{2},\left(T-t_{0}\right)^{2}\right\}>\max _{x \in \bar{\Omega}} \vartheta(x)+\delta, \quad 0<\beta<r_{0} .
$$

Therefore, by (6) and (8) the function $\psi(x, t)$ verifies the following properties

$$
\psi(x, 0) \leq \beta M-\delta, \quad \psi(x, T) \leq \beta M-\delta, \quad \text { for all } \quad x \in \bar{\Omega},
$$

there exists $\epsilon \in(0, T / 4)$ such that

$$
\max _{x \in \bar{\Omega}} \psi(x, t) \leq \beta M-\frac{\delta}{2}, \quad \text { for all } \quad t \in(0,2 \epsilon) \cup(T-2 \epsilon, T),
$$

and

$$
\min _{x \in \bar{\Omega}} \psi\left(x, t_{0}\right) \geq \beta M .
$$

We next introduce a function $\varphi: \bar{\Omega} \times \mathbb{R} \longrightarrow \mathbb{R}$ by

$$
\varphi(x, t)=e^{\gamma \psi(x, t)}:=\rho(x) \alpha(t), \quad \gamma>0,
$$

where $\gamma$ is a large parameter, $\rho(x)$ and $\alpha(t)$ are defined by

$\rho(x)=e^{\gamma\left(\left|x-x_{0}\right|^{2}+\beta M\right)} \geq e^{\gamma \beta M} \equiv d_{1}, \forall x \in \Omega$ and $\alpha(t)=e^{-\beta \gamma\left(t-t_{0}\right)^{2}} \leq 1, \forall t \in(0, T)$

and let

$$
\sigma \equiv \sigma(x, t)=s \gamma \varphi(x, t) .
$$

We use usual function spaces, $H^{k}(Q)$, and

$$
H^{2,1}(Q)=H^{1}\left(0, T ; L^{2}(\Omega)\right) \cap L^{2}\left(0, T ; H^{2}(\Omega)\right) .
$$

Let $(\mathbf{v}, y)$ a solution of the linear Biot consolidation system

$$
\begin{cases}\mathbf{v}_{t t}(x, t)-\Delta_{\mu, \lambda} \mathbf{v}(x, t)-\nabla\left(\lambda^{*} \operatorname{div} \mathbf{v}_{t}(x, t)\right)+\varrho_{1} \nabla y(x, t)=\mathbf{f}(x, t) & \text { in } Q, \\ y_{t}(x, t)-\Delta y(x, t)+\varrho_{2} \operatorname{div} \mathbf{v}_{t}(x, t)=h(x, t) & \text { in } Q,\end{cases}
$$

such that

$$
\begin{array}{ll}
\operatorname{Supp}(\mathbf{v}(\cdot, t)) \subset \Omega, \quad \operatorname{Supp}(y(\cdot, t)) \subset \Omega, & \text { for all } \quad t \in(0, T) . \\
\partial_{t}^{j} \mathbf{v}(x, 0)=\partial_{t}^{j} \mathbf{v}(x, T)=0, \quad y(x, 0)=y(x, T)=0 & \text { for all } \quad x \in \Omega, j=0,1 .
\end{array}
$$

The following theorem is a weighted Carleman estimate with second large parameter for Biot's consolidation system (15) with assumption (16). 
Theorem 2.1 (Carleman estimate) There exist two constants $\gamma_{*}>0$ and $C>0$ such that for any $\gamma>\gamma_{*}$, there exists $s_{*}=s_{*}(\gamma)>0$ such that the following estimate holds

$$
\begin{aligned}
& C \int_{Q}\left(\sigma\left|\nabla_{x, t} \mathbf{v}\right|^{2}+\sigma^{3}|\mathbf{v}|^{2}+\sigma^{4}|\operatorname{div} \mathbf{v}|^{2}+\sigma^{3}\left|\operatorname{div}_{t}\right|^{2}+\sigma^{2}|\nabla \operatorname{div} \mathbf{v}|^{2}+\sigma\left|\nabla \operatorname{div} \mathbf{v}_{t}\right|^{2}\right. \\
& \left.\quad+|\Delta y|^{2}+\sigma^{2}|\nabla y|^{2}+\sigma^{4}|y|^{2}\right) e^{2 s \varphi} d x d t \\
& \leq \int_{Q}\left(|\mathbf{f}|^{2}+|\nabla \mathbf{f}|^{2}+\gamma^{-1} \sigma|h|^{2}\right) e^{2 s \varphi} d x d t \\
& +\int_{Q}\left(\gamma^{-1}\left|\Delta \operatorname{divv}\left(x, t_{0}\right)\right|^{2}+\gamma^{-1}\left|\operatorname{div}_{t}\left(x, t_{0}\right)\right|^{2}\right. \\
& \left.\quad+\sigma^{4}\left|\operatorname{div} \mathbf{v}\left(x, t_{0}\right)\right|^{2}+\sigma^{2}\left|\nabla \operatorname{div} \mathbf{v}\left(x, t_{0}\right)\right|^{2}\right) e^{2 s \varphi} d x d t
\end{aligned}
$$

for any solution $(\mathbf{v}, y) \in H^{2}(Q) \times H^{2,1}(Q)$ to problem (15) which satisfy (16) and any $s \geq s_{*}$.

The proof is given by Bellassoued and Riahi [3].

\section{Proof of the main result}

This section is devoted to the proof of the Theorem 1.1. The idea of the proof is based on the Carleman estimate method in [3]. A usual methodology by [2, 3].

\subsection{Preliminaries estimate}

Lemma 3.1 Let $\omega$ be an open subdomain of $\Omega$ with regular boundary $\partial \omega \supset \Gamma$. There exists constants $\gamma_{*}, s_{*}$ and $C>0$ such that for any $s \geq s_{*}$ and any $\gamma \geq \gamma_{*}$ the following estimate holds :

$$
\int_{\omega \times(0, T)} \sigma^{4}|v|^{2} e^{2 s \varphi} d x d t \leq C \int_{\omega \times(0, T)} \sigma^{2}|\nabla v|^{2} e^{2 s \varphi} d x d t
$$

for any $v \in H^{1}(\omega \times(0, T))$ such that $v(x, t)=0$ on $\partial \omega \times(0, T)$.

Proof We multiply $\nabla v$ by $(\nabla \varphi) v e^{2 s \varphi}$ and using the divergence theorem, we obtain

$$
\begin{aligned}
\int_{\omega} \nabla v \cdot(\nabla \varphi) v e^{2 s \varphi} d x= & -\int_{\omega} v \operatorname{div}\left((\nabla \varphi) v e^{2 s \varphi}\right) d x \\
= & -\int_{\omega}|v|^{2} \Delta \varphi e^{2 s \varphi} d x-2 s \int_{\omega}|v|^{2}|\nabla \varphi|^{2} e^{2 s \varphi} d x \\
& -\int_{\omega} \nabla v \cdot(\nabla \varphi) v e^{2 s \varphi} d x .
\end{aligned}
$$

Therefore,

$$
\begin{aligned}
2 \int_{\omega} \sigma \nabla v \cdot(\nabla \vartheta) v e^{2 s \varphi} d x= & -2 \int_{\omega} \sigma^{2}|v|^{2}|\nabla \vartheta|^{2} e^{2 s \varphi} d x \\
& -\int_{\omega} \sigma|v|^{2} \Delta \vartheta e^{2 s \varphi} d x-\gamma \int_{\omega} \sigma|v|^{2}|\nabla \vartheta|^{2} e^{2 s \varphi} d x .
\end{aligned}
$$

Taking $\gamma \geq \gamma_{*}$ and $s \geq s_{*}$ sufficiently large, we obtain for any $\varepsilon>0$

$$
C \int_{\omega} \sigma^{2}|v|^{2} e^{2 s \varphi} d x \leq C_{\varepsilon} \int_{\omega}|\nabla v|^{2} e^{2 s \varphi} d x+\varepsilon \int_{\omega} \sigma^{2}|v|^{2} e^{2 s \varphi} d x .
$$


Integrating in $(0, T)$ and taking $\varepsilon$ small we obtain

$$
C \int_{\omega \times(0, T)} \sigma^{2}|v|^{2} e^{2 s \varphi} d x \leq C \int_{\omega \times(0, T)}|\nabla v|^{2} e^{2 s \varphi} d x .
$$

Applying the last inequality to $\sigma^{2} v$ we obtain

$$
\int_{\omega \times(0, T)} \sigma^{4}|v|^{2} e^{2 s \varphi} d x \leq C \int_{\omega \times(0, T)} \sigma^{2}|\nabla v|^{2} e^{2 s \varphi} d x
$$

for any $\gamma \geq \gamma_{*}$ and $s \geq s_{*}$. This completes the proof. Hence, by Lemma 3.1, we obtain the following Lemma.

Lemma 3.2 Let $(\mathbf{v}, y) \in H^{2}(Q) \times H^{2,1}(Q)$, satisfying

$$
\begin{cases}\mathbf{v}_{t t}-\Delta_{\mu, \lambda} \mathbf{v}-\nabla\left(\lambda^{*}(x) \operatorname{div} \mathbf{v}_{t}\right)+\varrho_{1}(x) \nabla y=\mathbf{f} & (x, t) \in Q \\ y_{t}-\Delta y+\varrho_{2}(x) \operatorname{div} \mathbf{v}_{t}=h & (x, t) \in Q \\ \mathbf{v}=0, \quad y=0 & (x, t) \in \Sigma\end{cases}
$$

and

$$
\partial_{t}^{j} \mathbf{v}(x, 0)=\partial_{t}^{j} \mathbf{v}(x, T)=0, \quad y(x, 0)=y(x, T)=0 \quad \text { for all } \quad x \in \Omega, j=0,1 .
$$

There exist positive constants $\gamma_{*}$ and $C>0$ such that, for any $\gamma \geq \gamma_{*}$ we can find $s_{*}$ and $D$, the following inequality holds

$$
\begin{gathered}
\int_{Q} e^{2 s \varphi}\left(\sigma\left|\nabla_{x, t} \mathbf{v}\right|^{2}+\sigma^{3}|\mathbf{v}|^{2}+\sigma^{4}|\operatorname{div} \mathbf{v}|^{2}+\sigma^{3}\left|\operatorname{div} \mathbf{v}_{t}\right|^{2}+\sigma^{2}|\nabla \operatorname{div} \mathbf{v}|^{2}\right. \\
\left.+\sigma\left|\nabla \operatorname{div} \mathbf{v}_{t}\right|^{2}+|\Delta y|^{2}+\sigma^{2}|\nabla y|^{2}+\sigma^{4}|y|^{2}\right) d x d t \leq C \int_{Q}\left(\gamma^{-1} \sigma|h|^{2}\right. \\
\left.+\sigma^{2}|\mathbf{f}|^{2}+|\nabla \mathbf{f}|^{2}\right) d x d t+C e^{D s}\left(\|\mathbf{v}\|_{H^{4}(\omega \times(0, T))}^{2}\right. \\
\left.+\left\|\mathbf{v}\left(\cdot, t_{0}\right)\right\|_{H^{3}(\Omega)}^{2}+\left\|\mathbf{v}_{t}\left(\cdot, t_{0}\right)\right\|_{H^{1}(\Omega)}^{2}\right)
\end{gathered}
$$

for any $s \geq s_{*}$.

Proof Let $\omega^{\prime} \subset \omega$ such that $\partial \omega^{\prime} \supset \Gamma$. In order to apply Carleman estimate, we introduce a cut-off function $\chi$ satisfying $0 \leq \chi \leq 1, \chi \in \mathscr{C}^{\infty}\left(\mathbb{R}^{3}\right), \chi=1$ in $\overline{\Omega \backslash \omega^{\prime}}$ and $\operatorname{supp} \chi \subset \Omega$. Put

$$
\widehat{\mathbf{v}}(x, t)=\chi(x) \mathbf{v}(x, t), \quad \widehat{y}(x, t)=\chi(x) y(x, t) .
$$

Noting that $(\widehat{\mathbf{v}}, \widehat{y}) \in H^{2}(Q) \times H^{2,1}(Q)$, and satisfying

$$
\begin{cases}\widehat{\mathbf{v}}_{t t}-\Delta_{\mu, \lambda} \widehat{\mathbf{v}}-\nabla\left(\lambda^{*} \operatorname{div} \widehat{\mathbf{v}}_{t}\right)+\varrho_{1} \nabla \widehat{y}=\widehat{\mathbf{f}} & (x, t) \in Q, \\ \widehat{y}_{t}-\Delta \widehat{y}+\varrho_{2} \operatorname{div} \widehat{\mathbf{v}}_{t}=\widehat{g} & (x, t) \in Q,\end{cases}
$$

with

$$
\operatorname{Supp}(\widehat{\mathbf{v}}(\cdot, t)) \subset \Omega \quad \operatorname{Supp}(\widehat{y}(\cdot, t)) \subset \Omega, \quad \forall t \in(0, T) .
$$

Here

$$
\begin{aligned}
\widehat{\mathbf{f}} & =\chi(x) \mathbf{f}(x, t)-\left[\Delta_{\mu, \lambda}, \chi\right] \mathbf{v}-\nabla\left(\lambda^{*} \nabla \chi \cdot \mathbf{v}_{t}\right)-\lambda^{*}(\nabla \chi) \operatorname{div} \mathbf{v}_{t}+\varrho_{1} y \nabla \chi \\
& \equiv \chi(x) \mathbf{f}(x, t)+P_{1} \mathbf{v}+Q_{1} \mathbf{v}_{t}+A_{0} y
\end{aligned}
$$




$$
\begin{aligned}
\widehat{g} & =\chi(x) h(x, t)-2 \nabla \chi \cdot \nabla y-y \Delta \chi+\varrho_{2} \nabla \chi \cdot \mathbf{v}_{t} \\
& \equiv \chi(x) h(x, t)+A_{1} y+Q_{0} \mathbf{v}_{t}
\end{aligned}
$$

and $P_{1}, Q_{1}, A_{1}$ are a first order partial differential operators with the coefficients are supported in $\omega^{\prime}$ and $A_{0}, Q_{0}$ are zeroth order partial diffrential operators supported also in $\omega^{\prime}$. Noting that $(\widehat{\mathbf{v}}, \widehat{y})$ satisfies (25), then we can apply the Carleman estimate for Biot's system (17) to $(\widehat{\mathbf{v}}, \widehat{y})$, we obtain

$$
\begin{aligned}
& C \int_{\left(\Omega \backslash \omega^{\prime}\right) \times(0, T)} e^{2 s \varphi}\left(\sigma\left|\nabla_{x, t} \mathbf{v}\right|^{2}+\sigma^{3}|\mathbf{v}|^{2}+\sigma^{4}|\operatorname{div} \mathbf{v}|^{2}+\sigma^{3}\left|\operatorname{div}_{t}\right|^{2}\right. \\
& \left.+\sigma^{2}|\nabla \operatorname{div} \mathbf{v}|^{2}+\sigma\left|\nabla \operatorname{div}_{t}\right|^{2}+|\Delta y|^{2}+\sigma^{2}|\nabla y|^{2}+\sigma^{4}|y|^{2}\right) d x d t \\
& \leq \int_{Q} e^{2 s \varphi}\left(\gamma^{-1} \sigma|\widehat{g}|^{2}+|\widehat{\mathbf{f}}|^{2}+|\nabla \widehat{\mathbf{f}}|^{2}\right) d x d t+\int_{Q}\left(\gamma^{-1}\left|\Delta \operatorname{div} \widehat{\mathbf{v}}\left(x, t_{0}\right)\right|^{2}\right. \\
& \left.+\gamma^{-1}\left|\operatorname{div} \widehat{\mathbf{v}}_{t}\left(x, t_{0}\right)\right|^{2}+\sigma^{4}\left|\operatorname{div} \widehat{\mathbf{v}}\left(x, t_{0}\right)\right|^{2}+\sigma^{2}\left|\nabla \operatorname{div} \widehat{\mathbf{v}}\left(x, t_{0}\right)\right|^{2}\right) e^{2 s \varphi} d x d t .
\end{aligned}
$$

Using the first equation of system (22) after taking divergence, we obtain the following estimate

$$
\begin{aligned}
\int_{\omega^{\prime} \times(0, T)}|\Delta y|^{2} e^{2 s \varphi} d x d t \leq & C e^{D s}\|\mathbf{v}\|_{H^{4}(\omega \times(0, T))}^{2} \\
& +\int_{\omega^{\prime} \times(0, T)}|\nabla y|^{2} e^{2 s \varphi} d x d t+\int_{Q} e^{2 s \varphi}|\nabla \mathbf{f}|^{2} d x d t .
\end{aligned}
$$

By the last inequality and (26), we deduce

$$
\begin{aligned}
& C \int_{\Omega \times(0, T)} e^{2 s \varphi}\left(\sigma|\nabla x, t \mathbf{v}|^{2}+\sigma^{3}|\mathbf{v}|^{2}+\sigma^{4}|\operatorname{div} \mathbf{v}|^{2}+\sigma^{3}\left|\operatorname{div} \mathbf{v}_{t}\right|^{2}+\sigma^{2}|\nabla \operatorname{div} \mathbf{v}|^{2}\right. \\
& \left.\quad+\sigma\left|\nabla \operatorname{div} \mathbf{v}_{t}\right|^{2}+|\Delta y|^{2}+\sigma^{2}|\nabla y|^{2}+\sigma^{4}|y|^{2}\right) d x d t \\
& \leq C \int_{Q}\left(\gamma^{-1} \sigma|h|^{2}+|\mathbf{f}|^{2}+|\nabla \mathbf{f}|^{2}\right) d x d t \\
& \quad+C\left(\int_{\omega^{\prime} \times(0, T)} \sigma^{4}|y|^{2} e^{2 s \varphi} d x d t+\int_{\omega^{\prime} \times(0, T)} \sigma^{2}|\nabla y|^{2} e^{2 s \varphi} d x d t\right) \\
& \quad+e^{D s}\left(\|\mathbf{v}\|_{H^{4}(\omega \times(0, T))}^{2}+\left\|\mathbf{v}\left(\cdot, t_{0}\right)\right\|_{H^{3}(\Omega)}^{2}+\left\|\mathbf{v}_{t}\left(\cdot, t_{0}\right)\right\|_{H^{1}(\Omega)}^{2}\right) .
\end{aligned}
$$

Let $\chi_{1}$ be a cut-off function satisfying $0 \leq \chi_{1} \leq 1, \chi_{1} \in \mathscr{C}^{\infty}\left(\mathbb{R}^{3}\right), \chi_{1}=1$ in $\overline{\omega^{\prime}}$ and $\operatorname{Supp}\left(\chi_{1}\right) \subset \omega$. Let us consider $z(x, t)=\chi_{1}(x) y(x, t) \in H^{1}(\omega \times(0, T))$ and $z(x, t)=0$ for all $(x, t) \in \partial \omega \times(0, T)$, so that by Lemma 3.1, we have

$$
\begin{aligned}
& \int_{\omega^{\prime} \times(0, T)} \sigma^{4}|y|^{2} \sigma e^{2 s \varphi} d x d t \leq \int_{\omega \times(0, T)} \sigma^{4}|z|^{2} e^{2 s \varphi} d x d t \\
& \leq C \int_{\omega \times(0, T)} \sigma^{2}|\nabla y|^{2} e^{2 s \varphi} d x d t+C \int_{Q} \sigma^{2}|y|^{2} e^{2 s \varphi} d x d t .
\end{aligned}
$$

Furthermore by the first equation of (22), we have

$$
\int_{\omega^{\prime} \times(0, T)} \sigma^{2}|\nabla y|^{2} e^{2 s \varphi} d x d t \leq C e^{D s}\|\mathbf{v}\|_{H^{3}(\omega \times(0, T))}^{2}
$$




$$
+\int_{\Omega \times(0, T)} \sigma^{2}|\mathbf{f}|^{2} e^{2 s \varphi} d x d t
$$

Inserting (28) and (29) in (27), we obtain (24). This completes the proof of the Lemma.

Let $(\mathbf{v}, y) \in H^{2}(Q) \times H^{2,1}(Q)$ satisfying

$$
\begin{array}{ll}
\mathbf{v}_{t t}-\Delta_{\mu, \lambda} \mathbf{v}-\nabla\left(\lambda^{*} \operatorname{div} \mathbf{v}_{t}\right)+\varrho_{1} \nabla y=\mathbf{f} & (x, t) \in Q \\
y_{t}-\Delta y+\varrho_{2} \operatorname{div} \mathbf{v}_{t}=h & (x, t) \in Q \\
\mathbf{v}=0, y=0 & (x, t) \in \Sigma
\end{array}
$$

Put

$$
\widetilde{\mathbf{v}}(x, t)=\eta(t) \mathbf{v}(x, t), \quad \widetilde{y}(x, t)=\eta(t) y(x, t) .
$$

Noting that $(\widetilde{\mathbf{v}}, \widetilde{y}) \in H^{2}(Q) \times H^{2,1}(Q)$ satisfies

$$
\begin{array}{ll}
\widetilde{\mathbf{v}}_{t t}-\Delta_{\mu, \lambda} \widetilde{\mathbf{v}}-\nabla\left(\lambda^{*} \operatorname{div} \widetilde{\mathbf{v}}_{t}\right)+\varrho_{1} \nabla \widetilde{y}=\eta \mathbf{f}+\eta_{t t} \mathbf{v}+2 \eta_{t} \mathbf{v}_{t}-\eta_{t} \nabla\left(\lambda^{*} \operatorname{div} \mathbf{v}\right) & (x, t) \in Q, \\
\widetilde{y}_{t}-\Delta \widetilde{y}+\varrho_{2} \operatorname{div} \widetilde{\mathbf{v}}_{t}=\eta h+\eta_{t}\left(y-\varrho_{2} \operatorname{div} \mathbf{v}\right) & (x, t) \in Q, \\
\widetilde{\mathbf{v}}=0, \widetilde{y}=0 & (x, t) \in \Sigma,
\end{array}
$$

Henceforth we fix $\gamma>0$ sufficiently large. By $N_{s, \varphi}$ we denote the quantity

$$
\begin{aligned}
& N_{s, \varphi}(\mathbf{v}, y)=\int_{Q} e^{2 s \varphi}\left(s\left|\nabla_{x, t} \mathbf{v}\right|^{2}+s^{3}|\mathbf{v}|^{2}+s^{4}|\operatorname{div} \mathbf{v}|^{2}+s^{3}\left|\operatorname{div}_{t}\right|^{2}\right. \\
& \left.+s^{2}|\nabla \operatorname{div} \mathbf{v}|^{2}+s\left|\nabla \operatorname{div} \mathbf{v}_{t}\right|^{2}+|\Delta y|^{2}+s^{2}|\nabla y|^{2}+s^{4}|y|^{2}\right) d x d t
\end{aligned}
$$

We introduce a cut-off function $\eta$ satisfying $0 \leq \eta \leq 1, \eta \in \mathcal{C}^{\infty}(\mathbb{R}), \eta=1$ in $(2 \varepsilon, T-2 \varepsilon)$ and $\operatorname{Supp}(\eta) \subset(\varepsilon, T-\varepsilon)$. Finally we denote

$$
\widetilde{\mathbf{v}}=\eta \mathbf{v}, \quad \widetilde{y}=\eta y .
$$

Setting $d_{0}=e^{(\beta M-\delta / 2) \gamma}$, by (9), we have

$$
\max _{x \in \Omega} \varphi(x, t) \leq d_{0}, \quad t \in(0,2 \varepsilon) \cup(T-2 \varepsilon, T) .
$$

We have the following lemma :

Lemma 3.3 There exist three positive constants $s_{*}, C>0$ and $D$ such that the following inequality holds :

$$
\begin{aligned}
& C N_{s, \varphi}(\widetilde{\mathbf{v}}, \widetilde{y}) \leq \int_{Q}\left(s|h|^{2}+s^{2}|\mathbf{f}|^{2}+|\nabla \mathbf{f}|^{2}\right) e^{2 s \varphi} d x d t \\
& +e^{D s}\left(\|\mathbf{v}\|_{H^{4}(\omega \times(0, T))}^{2}+\left\|\mathbf{v}\left(\cdot, t_{0}\right)\right\|_{H^{3}(\Omega)}^{2}+\left\|\mathbf{v}_{t}\left(\cdot, t_{0}\right)\right\|_{H^{1}(\Omega)}^{2}\right) \\
& +C s^{2} e^{2 d_{0} s}\left(\|\mathbf{v}\|_{H^{1}\left(0, T ; H^{2}(\Omega)\right)}^{2}+\|y\|_{L^{2}(Q)}^{2}\right),
\end{aligned}
$$

for any $s \geq s_{*}$ and any $(\widetilde{\mathbf{v}}, \widetilde{y}) \in H^{2}(Q) \times H^{2,1}(Q)$ satisfying (30).

Proof Applying Carleman estimate (24) to $(\widetilde{\mathbf{v}}, \widetilde{y})$, we obtain 


$$
\begin{gathered}
C N_{s, \varphi}(\widetilde{\mathbf{v}}, \widetilde{y}) \leq \int_{Q}\left(s|h|^{2}+s^{2}|\mathbf{f}|^{2}+|\nabla \mathbf{f}|^{2}\right) e^{2 s \varphi} d x d t \\
+e^{D s}\left(\|\mathbf{v}\|_{H^{4}(\omega \times(0, T))}^{2}+\left\|\mathbf{v}\left(\cdot, t_{0}\right)\right\|_{H^{3}(\Omega)}^{2}+\left\|\mathbf{v}_{t}\left(\cdot, t_{0}\right)\right\|_{H^{1}(\Omega)}^{2}\right) \\
+\int_{Q}\left(s^{2}\left(\left|\eta_{t t}\right|^{2}+\left|\eta_{t}\right|^{2}\right)\left(|y|^{2}+|\mathbf{v}|^{2}+\left|\mathbf{v}_{t}\right|^{2}+|\nabla \mathbf{v}|^{2}+\left|\nabla \mathbf{v}_{t}\right|^{2}+|\nabla(\operatorname{div} \mathbf{v})|^{2}\right) e^{2 s \varphi} d x d t\right.
\end{gathered}
$$

for any $\gamma \geq \gamma_{*}$ and $s \geq s_{*}$. Since $\operatorname{Supp}\left(\eta_{t t}\right), \operatorname{Supp}\left(\eta_{t}\right) \subset(0,2 \epsilon) \cup(T-2 \epsilon, T)$, we obtain from (32)

$$
\begin{aligned}
& \int_{Q}\left(s^{2}\left(\left|\eta_{t t}\right|^{2}+\left|\eta_{t}\right|^{2}\right)\left(|y|^{2}+|\mathbf{v}|^{2}+\left|\mathbf{v}_{t}\right|^{2}+|\nabla \mathbf{v}|^{2}+\left|\nabla \mathbf{v}_{t}\right|^{2}+|\nabla(\operatorname{div} \mathbf{v})|^{2}\right) e^{2 s \varphi} d x d t\right. \\
& \leq C s^{2} e^{2 d_{0} s}\left(\|\mathbf{v}\|_{H^{1}\left(0, T ; H^{2}(\Omega)\right)}^{2}+\|y\|_{L^{2}(Q)}^{2}\right) .
\end{aligned}
$$

This completes the proof of the lemma.

\subsection{Completion of the proof of the main result}

Consider now the following system

$$
\begin{array}{ll}
\mathbf{u}_{t t}-\Delta_{\mu, \lambda} \mathbf{u}-\nabla\left(\lambda^{*} \operatorname{div} \mathbf{u}_{t}\right)+\varrho_{1} \nabla \theta=0 & (x, t) \in Q \\
\theta_{t}-\Delta \theta+\varrho_{2} \operatorname{div} \mathbf{u}_{t}=g & (x, t) \in Q \\
\mathbf{u}=0, \theta=0 & (x, t) \in \Sigma
\end{array}
$$

where the heat source term $g$ is given by

$$
g(x, t)=q(x) k(x, t) .
$$

Let $\mathbf{v}=u_{t}$ and $y=\theta_{t}$. Then, we have

$$
\begin{array}{ll}
\mathbf{v}_{t t}-\Delta_{\mu, \lambda} \mathbf{v}-\nabla\left(\lambda^{*} \operatorname{div} \mathbf{v}_{t}\right)+\varrho_{1} \nabla y=0 & (x, t) \in Q \\
y_{t}-\Delta y+\varrho_{2} \operatorname{div} \mathbf{v}_{t}=g_{t} & (x, t) \in Q \\
\mathbf{v}=0, y=0 & (x, t) \in \Sigma
\end{array}
$$

We apply Lemma 3.3 to $(\widetilde{\mathbf{v}}, \widetilde{y})$ solution of the following system

$$
\begin{array}{ll}
\widetilde{\mathbf{v}}_{t t}-\Delta_{\mu, \lambda} \widetilde{\mathbf{v}}-\nabla\left(\lambda^{*} \operatorname{div} \widetilde{\mathbf{v}}_{t}\right)+\varrho_{1} \nabla \widetilde{y}=\eta_{t t} \mathbf{v}+2 \eta_{t} \mathbf{v}_{t}-\eta_{t} \nabla\left(\lambda^{*} \operatorname{div} \mathbf{v}\right) & (x, t) \in Q, \\
\widetilde{y}_{t}-\Delta \widetilde{y}+\varrho_{2} \operatorname{div} \widetilde{\mathbf{v}}_{t}=\eta g_{t}+\eta_{t}\left(y-\varrho_{2} \operatorname{div} \mathbf{v}\right) & (x, t) \in Q, \\
\widetilde{\mathbf{v}}=0, \widetilde{y}=0 & (x, t) \in \Sigma,
\end{array}
$$

we obtain the following estimate :

$$
\begin{aligned}
& C N_{s, \varphi}(\widetilde{\mathbf{v}}, \widetilde{y}) \leq \int_{Q} s\left|g_{t}\right|^{2} e^{2 s \varphi} d x d t \\
& +e^{D s}\left(\|\mathbf{v}\|_{H^{4}(\omega \times(0, T))}^{2}+\left\|\mathbf{v}\left(\cdot, t_{0}\right)\right\|_{H^{3}(\Omega)}^{2}+\left\|\mathbf{v}_{t}\left(\cdot, t_{0}\right)\right\|_{H^{1}(\Omega)}^{2}\right) \\
& +C s^{2} e^{2 d_{0} s}\left(\|\mathbf{v}\|_{H^{1}\left(0, T ; H^{2}(\Omega)\right)}^{2}+\|y\|_{L^{2}(Q)}^{2}\right)
\end{aligned}
$$

for sufficiently large $s>0$.

We have the following Lemma 
Lemma 3.4 There exists a positive constant $C>0$ such that the following estimate

$$
\int_{\Omega}\left|z\left(x, t_{0}\right)\right|^{2} d x \leq C \int_{Q}\left(s|z(x, t)|^{2}+s^{-1}\left|z_{t}(x, t)\right|^{2}\right) d x d t,
$$

for any $z \in H^{1}\left(0, T ; L^{2}(\Omega)\right)$.

Proof By direct computations, we have

$$
\begin{aligned}
\int_{\Omega} \eta^{2}\left(t_{0}\right)\left|z\left(x, t_{0}\right)\right|^{2} d x & =\int_{0}^{t_{0}} \frac{d}{d t}\left(\int_{\Omega} \eta^{2}(t)|z(x, t)|^{2} d x\right) d t \\
& =2 \int_{0}^{t_{0}} \int_{\Omega} \eta^{2}(t) z(x, t) z_{t}(x, t) d x d t+2 \int_{0}^{t_{0}} \int_{\Omega} \eta_{t}(t) \eta(t)|z(x, t)|^{2} d x d t .
\end{aligned}
$$

Then, we have

$$
\int_{\Omega}\left|z\left(x, t_{0}\right)\right|^{2} d x \leq C \int_{Q}\left(s|z(x, t)|^{2}+s^{-1}\left|z_{t}(x, t)\right|^{2}\right) d x d t .
$$

This complete the proof of the lemma.

Second, we apply lemma 3.4 to $y_{1}(x, t)=\eta(t) e^{2 s \varphi(x, t)} y(x, t)=e^{2 s \varphi(x, t)} \widetilde{y}(x, t)$ and by the second equation of (35), we obtain

$$
\begin{aligned}
& \int_{\Omega} e^{2 s \rho(x)}\left|y\left(x, t_{0}\right)\right|^{2} d x \leq \int_{Q} \sigma e^{2 s \varphi}|\widetilde{y}(x, t)|^{2} d x d t+\int_{Q} \sigma^{-1} e^{2 s \varphi}\left|\widetilde{y}_{t}(x, t)\right|^{2} d x d t \\
& \leq \int_{Q} \sigma e^{2 s \varphi}|\widetilde{y}(x, t)|^{2} d x d t+\int_{Q} \sigma^{-1} e^{2 s \varphi}\left|g_{t}(x, t)\right|^{2} d x d t \\
& +\int_{Q} \sigma^{-1} e^{2 s \varphi}|\Delta \widetilde{y}(x, t)|^{2} d x d t+\int_{Q} \sigma^{-1} e^{2 s \varphi}\left|\operatorname{div} \widetilde{\mathbf{v}}_{t}\right|^{2} d x d t \\
& +\int_{Q} \sigma^{-1} e^{2 s \varphi}\left|\eta_{t}\right|^{2}\left(|y|^{2}+|\operatorname{div} \mathbf{v}|^{2}\right) d x d t \\
& \leq \int_{Q} \sigma e^{2 s \varphi}|\widetilde{y}(x, t)|^{2} d x d t+\int_{Q} \sigma^{-1} e^{2 s \varphi}\left|g_{t}(x, t)\right|^{2} d x d t \\
& +\int_{Q} \sigma^{-1} e^{2 s \varphi}|\Delta \widetilde{y}(x, t)|^{2} d x d t+\int_{Q} \sigma^{-1} e^{2 s \varphi}\left|\operatorname{div} \widetilde{\mathbf{v}}_{t}\right|^{2} d x d t \\
& +e^{2 s d_{0}}\left(\|\mathbf{v}\|_{H^{1}(Q)}^{2}+\|y\|_{L^{2}(Q)}^{2}\right) .
\end{aligned}
$$

Then,

$$
\begin{aligned}
& C s \int_{\Omega} e^{s \rho(x)}\left|y\left(x, t_{0}\right)\right|^{2} d x \leq \int_{Q} e^{2 s \varphi}\left|g_{t}(x, t)\right|^{2} d x d t \\
& +\int_{Q} e^{2 s \varphi}\left(|\Delta \widetilde{y}|^{2}+\sigma^{2}|\widetilde{y}|^{2}+\left|\operatorname{div} \widetilde{\mathbf{v}_{t}}\right|^{2}\right) d x d t+s e^{2 s d_{0}}\left(\|\mathbf{v}\|_{H^{1}(Q)}^{2}+\|y\|_{L^{2}(Q)}^{2}\right) \\
& \leq \int_{Q} e^{2 s \varphi}\left|g_{t}(x, t)\right|^{2} d x d t+C \mathcal{N}_{s, \varphi}(\widetilde{\mathbf{v}}, \widetilde{y})+s e^{2 s d_{0}}\left(\|\mathbf{v}\|_{H^{1}(Q)}^{2}+\|y\|_{L^{2}(Q)}^{2}\right) .
\end{aligned}
$$

Moreover, using (36), we get

$$
s \int_{\Omega} e^{2 s \rho(x)}\left|y\left(x, t_{0}\right)\right|^{2} d x \leq C s \int_{Q} e^{2 s \varphi}\left|g_{t}(x, t)\right|^{2} d x d t
$$




$$
\begin{aligned}
& +e^{D s}\left(\|\mathbf{v}\|_{H^{4}(\omega \times(0, T))}^{2}+\left\|\mathbf{v}\left(., t_{0}\right)\right\|_{H^{3}(\Omega)}^{2}+\left\|\mathbf{v}_{t}\left(., t_{0}\right)\right\|_{H^{1}(\Omega)}^{2}\right) \\
& +C s^{2} e^{2 s d_{0}}\left(\|\mathbf{v}\|_{H^{1}\left(0, T ; H^{2}(\Omega)\right)}^{2}+\|y\|_{L^{2}(Q)}^{2}\right) .
\end{aligned}
$$

On the one hand, by the second equation in (33), we have

$$
y\left(x, t_{0}\right)=\Delta \theta\left(x, t_{0}\right)-\varrho_{2} \operatorname{div} \mathbf{v}\left(x, t_{0}\right)+q(x) k\left(x, t_{0}\right) .
$$

Moreover, we have

$$
|q(x)| \leq|q(x)|\left|k\left(x, t_{0}\right)\right| \leq\left|y\left(x, t_{0}\right)\right|+\left|\Delta \theta\left(x, t_{0}\right)\right|+\left|\operatorname{div} \mathbf{v}\left(x, t_{0}\right)\right| .
$$

Then,

$$
\begin{aligned}
& \int_{\Omega} e^{2 s \rho(x)}|q(x)|^{2} d x \leq \int_{\Omega} e^{2 s \rho(x)}\left|y\left(x, t_{0}\right)\right|^{2} d x \\
& +\int_{\Omega} e^{2 s \rho(x)}\left|\operatorname{div} \mathbf{v}\left(x, t_{0}\right)\right|^{2} d x+\int_{\Omega} e^{2 s \rho(x)}\left|\Delta \theta\left(x, t_{0}\right)\right|^{2} d x .
\end{aligned}
$$

We deduce that

$$
\begin{aligned}
& s \int_{\Omega} e^{2 s \rho(x)}|q(x)|^{2} d x \leq s \int_{\Omega} e^{2 s \rho(x)}\left|y\left(x, t_{0}\right)\right|^{2} d x \\
& +s \int_{\Omega} e^{2 s \rho(x)}\left|\operatorname{divv}\left(x, t_{0}\right)\right|^{2} d x+C e^{s D}\left\|\Delta \theta\left(x, t_{0}\right)\right\|_{L^{2}(\Omega)}^{2} .
\end{aligned}
$$

Inserting (39) into (43), we get

$$
\begin{aligned}
& s \int_{\Omega} e^{2 s \rho(x)}|q(x)|^{2} d x \leq C s \int_{Q} e^{2 s \varphi}\left|g_{t}(x, t)\right|^{2} d x d t+C s^{2} e^{2 s d_{0}} M_{0}^{2} \\
& +C e^{D s}\left(\|\mathbf{v}\|_{H^{4}(\omega \times(0, T))}^{2}+\left\|\mathbf{v}\left(., t_{0}\right)\right\|_{H^{3}(\Omega)}^{2}\right. \\
& \left.+\left\|\mathbf{v}_{t}\left(., t_{0}\right)\right\|_{H^{1}(\Omega)}^{2}+\left\|\Delta \theta\left(x, t_{0}\right)\right\|_{L^{2}(\Omega)}^{2}\right) .
\end{aligned}
$$

Selecting $\kappa \in\left(d_{0}, d_{1}\right)$ such that $C s^{2} e^{2 s d_{0}} \leq e^{2 \kappa s}$ for any $s$ large, we get

$$
\begin{aligned}
& s \int_{\Omega} e^{2 s \rho(x)}|q(x)|^{2} d x \leq C s \int_{Q} e^{2 s \varphi}\left|g_{t}(x, t)\right|^{2} d x d t+e^{2 \kappa s} M_{0}^{2} \\
& +C e^{D s}\left(\|\mathbf{v}\|_{H^{4}(\omega \times(0, T))}^{2}+\left\|\mathbf{v}\left(., t_{0}\right)\right\|_{H^{3}(\Omega)}^{2}\right. \\
& \left.+\left\|\mathbf{v}_{t}\left(., t_{0}\right)\right\|_{H^{1}(\Omega)}^{2}+\left\|\theta\left(., t_{0}\right)\right\|_{H^{2}(\Omega)}^{2}\right) .
\end{aligned}
$$

Now we return to the first integral term in $Q$ on the right-hand side term of (45).

$$
\int_{Q} e^{2 s \varphi}\left|q(x) k_{t}\right|^{2} d x d t \leq \int_{\Omega} e^{2 s \rho(x)}|q(x)|^{2}\left(\int_{0}^{T} e^{-2 s(\rho-\varphi)}\left\|k_{t}(., t)\right\|_{L^{\infty}(\Omega)}^{2} d t\right) d x .
$$

By the Lebesgue theorem, we obtain

$$
\int_{0}^{T} e^{-2 s(\rho-\varphi)}\left\|k_{t}(., t)\right\|_{L^{\infty}(\Omega)}^{2} d t=\int_{0}^{T} e^{-2 s \rho(x)(1-\alpha(t))}\left\|k_{t}(., t)\right\|_{L^{\infty}(\Omega)}^{2} d t
$$




$$
\leq \int_{0}^{T} e^{-2 s(1-\alpha(t))}\left\|k_{t}(., t)\right\|_{L^{\infty}}^{2} d t=o(1),
$$

as $s \rightarrow \infty$. By (45), we have

$$
\begin{aligned}
& s\left\|e^{s \rho} q\right\|_{L^{2}(\Omega)}^{2} \leq o(1) \int_{Q} s e^{2 s \rho}|q(x)|^{2} d x d t+e^{2 \kappa s} M_{0}^{2} \\
& +C e^{D s}\left(\|\mathbf{v}\|_{H^{4}(\omega \times(0, T))}^{2}+\left\|\mathbf{v}\left(., t_{0}\right)\right\|_{H^{3}(\Omega)}^{2}\right. \\
& \left.+\left\|\mathbf{v}_{t}\left(., t_{0}\right)\right\|_{H^{1}(\Omega)}^{2}+\left\|\theta\left(., t_{0}\right)\right\|_{H^{2}(\Omega)}^{2}\right) .
\end{aligned}
$$

for all $s \geq s_{*}$.

On the other hand, using the fact that $\rho(x) \geq d_{1}$ for all $x \in \Omega$. Then for sufficiently large $s>0$, we have

$$
\begin{aligned}
& \|q\|_{L^{2}(\Omega)}^{2} \leq C e^{\gamma_{1} s}\left(\|\mathbf{v}\|_{H^{4}(\omega \times(0, T))}^{2}+\left\|\mathbf{v}\left(., t_{0}\right)\right\|_{H^{3}(\Omega)}^{2}\right. \\
& \left.+\left\|\mathbf{v}_{t}\left(., t_{0}\right)\right\|_{H^{1}(\Omega)}^{2}+\left\|\theta\left(., t_{0}\right)\right\|_{H^{2}(\Omega)}^{2}\right)+C e^{-\gamma_{2} s} M_{0}^{2}
\end{aligned}
$$

Finally, minimizing the right hand side with respect to $s$, we obtain

$$
\|q\|_{L^{2}(\Omega)} \leq C\left(\|\mathbf{v}\|_{H^{4}(\omega \times(0, T))}+\left\|\theta\left(., t_{0}\right)\right\|_{H^{2}(Q)}+\left\|\mathbf{v}\left(., t_{0}\right)\right\|_{H^{3}(\Omega)}+\left\|\mathbf{v}_{t}\left(., t_{0}\right)\right\|_{H^{1}(\Omega)}\right)^{\delta} .
$$

Thus proof of Theorem 1.1 is completed.

\section{Bibliographie}

[1] H. BarucQ, M.Madaune-Tort And P. SAint-Macary, « Theoretical aspects of wave propagation for Biot's consolidation problem », Monografías del Seminario Mathemático García de Galdeano, vol. 31, 2004.

[2] M.Bellassoued, M. Yamamoto, «Carleman estimates and inverse heat source problem for thermoelasticity system », Inverse problems, vol. 27, 2011.

[3] M.Bellassoued, B. RiAhi « Carleman estimate for Biot consolidation system in poroelasticity and application to inverse problem », Mathematical Method in the Applied Sciences, 2016.

[4] B. WU, J. LIU, « Conditional stability and uniqueness for determining two coefficients in a hyperbolic-parabolic system », Journal, vol. 27, 2011.

[5] J-L.Lions, E.MAGENES, « Non-homogenous boundary value problems and applications », Springer-Verlag, vol. I and II, 1972. 